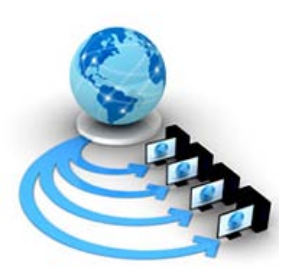

\title{
FRAMEWORK FOR CERTIFICATION OF REUSABLE SOFTWARE COMPONENTS
}

\author{
Anshul Kalia \\ Ph.D. Scholar, Dept. of RIC, \\ IK Gujral Punjab Technical University, \\ Kapurthala, India
}

\author{
Sumesh Sood \\ HOD, Dept. of Computer Applications, \\ IK Gujral Punjab Technical University Campus Dinanagar, \\ Dinanagar, India.
}

\begin{abstract}
Certification is a technique that plays a vital role in endorsing software components. It helps in increasing the importance of software components. It tries to validate the quality of components and makes it reliable for reuse. This increases the probability of software components to be reused. The study presented the framework for certification of reusable software components. While presenting the framework the study stated about the certifiable artifacts and certifying authority. It also stated the methods of certification, certification practices to be followed, and the certification services. The certification process actually ratifies the quality of software components. The certification methods involve the request for certification, processing the request, and issuance of certificates. The certification practices are categorized into 'for reuse' and 'with reuse'. The certification services stated about the services provided in relation to certificates. It is easy to rely on products with valid certificates. The software components without certification do not guarantee the quality aspects. It may then be able to compromise the quality of software products, which in turn, can vitiate the reliability of software components and consequently, the software products.

Keywords: Certification, certifiable artifacts, certifying authority, issuance, renewal.
\end{abstract}

\section{INTRODUCTION}

Certification of reusable software components is a technique that assures the users about the quality and reliability of the reusable software components [1] [2]. Certification is a kind of technique that is being used in every walk of life. It is being used to certify the things, their quality, and certain qualitative aspects of human beings are also certified by implying different methods. It assures the user that the product or the human possesses the quality aspect as certified by the certifying authority. Certification acts as a proof of validity to the quality of a person or a product. Certification makes one to easily rely on the quality and integrity of a product [3]. When a certificate is attached with a product, its chances of sale increased. If we take an example of a network engineer possessing a certificate of 'certified network professional', his chances of being hired for that job increases instead of the ones without the certificates [4]. The same case applies to the reusable software components. If the certified reusable software components are there, then their chances of being reused will be increased. Also this will help to create a sense of reliability amongst the users of the reusable components.

The certification of reusable software components advocates, that, the software components which are being developed for reuse should be certified through an independent certifying authority [5] [6]. When there should be the components with valid certificates, then it would be easy for the users to rely on the quality and security aspects of the components. Certification acts as a factor to increase the reliability of reusable software components. The area of component certification has not been explored to that potential or extent, as it has to be. It is still lacking the formal methods of certification, absence of independent certification authority, unspecified certification process and practices etc [7]. If a certification system for reusable components is put in place, then, it will help to standardize the process of reusable component development, improve the quality, and increased reliability of software components available for reuse. This will certainly help to streamline the segment of reusable software components. With increased quality and reliability, the segment will flourish more. This section of study tries to carve out a standardized mechanism that should help in certifying the reusable components.

\section{CERTIFICATION FRAMEWORK}

The certification framework will help to achieve accuracy, quality, competence, and reliability amongst the reusable software components. The framework states the methods of certification, certifiable artifacts, the certification authority, and the practices to be followed [1] [3]. It is felt that the certificate should be such an entity that it may be executed and understood by the machine, it may be transacted along with the component itself. For this, it is required to adopt the public key infrastructure (PKI) [5] [6].

\section{A. Certifiable Artifacts}

The term certifiable artifacts define the items or the micro components of a reusable component, which can be certified. The certifiable artifacts may include the source code of component, various documents of component such as requirement specifications document, design description, implementation details, deployment details, test plans, test cases, maintenance plans, maintenance log, and others etc. The artifacts mentioned here tell that, these artifacts for reusable components can be certified as an individual item or as part of whole component. A certificate of a certified component or a part of component can also be considered as the certifiable artifact [1]. 


\section{B. Certifying Authority (CA)}

The certifying authority refers to an independent authority that has the capability and expertise to certify the certifiable artifacts of a reusable component. The certifying authority is the one responsible for certifying the reusable components. It may be an organization in general that should certify the components. As of now, the area of reusable software components lacks such a certifying authority, which may provide certificates to the components concerning the value of their claimed property. Though there exist some certification authorities, but they are generally concerned about the security aspects of web browsers or the security aspects of the software which are being transacted over the internet. They are not concerned much about the quality aspect of software. This study feels the requirement of such an authority that should be able to address the security as well as the quality aspects of reusable software components. Also there is a possibility that the existing certifying authorities can club the security aspects with the quality aspect as and when required to certify the components. This step can prove to be a vital for the market related to reusable components. Also it will help to curb the need to introduce a new certifying authority. The existing certifying authority with enhanced facility can fill the gap [5] [6].

\section{Methods of Certification}

The certification method defines the way by which the reusable software components may be certified. The methods of certification become more significant, when it is to be implied, when developing component for reuse. In case of development with reuse, it is advised that one should make use of certified components [8]. When a software component is being developed for reuse, it is required to be certified. There may be two approaches that can be followed: certification right from the inception of development process, the second one is certification after the complete development. It is the responsibility of the developer to get its component certified. For this, it is required to make request to the certifying authority for certification. Once the request is made, it is up to the certifying authority to take over the processions. The methods that should be adopted for certification of reusable components are defined as follows [9] [10]:

\section{1) Application For Certification:}

- An application for certification of reusable software components is to be sent to the certifying authority by the developer of the component.

- The application should be sent in a method specified by the certifying authority.

- $\quad$ The applications should be sent through proper channel as stated by the certifying authority.

- It should be made clear by CA that the applications sent through unspecified means should be rejected without any considerations.

- An acknowledgement on acceptance of application for processing should be sent to the applicant.
The applicant is also required to pay the applicable fees as specified by the CA while submitting the application.

2) Processing Certification Application:

- On receiving the application for certification, it is now the turn of certifying authority to get into the action.

- The CA will collect information regarding the identity and authentication of the applicant.

- It will collect the requisites for processing the application from the applicant.

- That is, it will collect the supporting information as required for analyzing the application.

- It will give applicant the estimated time required to process the application.

- It will then start the process of analyzing the component for its claimed property by vetting the supporting information.

- Once the process of analysis is complete, the CA will then take the decision of accepting or rejecting the component for the issuance of certificate.

- The decision, whatever it may be, should be communicated to the applicant about the acceptance or rejection of the component for certificate.

3) Issuing Certificate:

- If the component is accepted, a formal certificate is to be issued certifying the reusable component by the CA.

- The CA should also notify the applicant about the issuance of certificate.

- The certificate certifying the claimed property of the component should then be published and be sent to the applicant.

- The certificate should be provided in a form such as the public key infrastructure (PKI), so that it can be attached digitally along with the reusable component [9].

\section{Certification Practices}

The certification practices state how the component is to be certified by using the certification methods. It also states the services provided under the certification lifecycle. The certification practices are considered from two view points, i.e., development for reuse and development with reuse [9].

\section{1) Development For Reuse:}

In this case, the component being developed for reuse is to get certified. While certifying such a reusable component, one of the two approaches can be followed. These are:

i. Certification Since Inception

- It means that the process of certification should be introduced right after the inception of development process of component for reuse.

- In this process, when the development of the component for reuse starts, the developer induces the CA by making a request for certification.

- The CA takes charge of the processions, and start analyzing the ongoing development process. 
- It also analyses the previously completed development process of component, that is, the development details of component before making it the component for reuse.

- The analysis process initiated by CA will complete, when the development process of component for reuse ends.

- After the analysis process, the CA will make decisions on the basis of analysis conducted.

- The decision will be either to accept the component for the award of certificate or to reject the component.

- The decision will then be communicated to the developer or the applicant, who had made a request for the certification of component.

- Finally, the certificate will be issued and be published.

ii. Certification After Completion

- It means that the process of certification should be introduced after the process of developing a component for reuse has been completed.

- In this process, the CA is induced for certification by making a request for certification, when the development process of component for reuse is complete.

- The CA will then ask the applicant to provide the requisite data and information that helps in deciding about the acceptance or rejection of component for the award of certificate.

- The data will be analyzed, and the decision will be taken about the acceptance or rejection of component.

- The decision taken will be communicated to the applicant.

- If the component is accepted for award of certification, the certificate will be issued to the applicant and will be published.

2) Development With Reuse:

In this case, a reusable component that is being used for developing other new software is to get certified.

- Firstly, it is advised that the user of reusable component should make use of components that has already been certified through a CA.

- Using the reusable components that are not certified can be risky and unsafe as far as the security is concerned.

- Also it can compromise the quality of product being developed, if the component being reused does not conforms to quality standards.

- In such a scenario, if the request is made to certify a reusable component that is being reused for development of another product, the CA will start analysis after the request is made.

- The CA will collect the identity and authentication details about the applicant.

- It will require the supporting data and information about the component to be certified.
- Upon analysis of concerned data and information, the CA will make decision about the acceptance or rejection of component.

- The decision about the acceptance or rejection will be intimated to the applicant.

- The certificate will be issued and published, if accepted for award of certificate.

\section{E. Certification Services}

The certification services include the practices that are followed after the decision about the acceptance or rejection of component for the award of certification. These services may include the following [1]:

\section{1) Certificate Construction:}

During certificate construction, a certificate will be created. The created certificate will contain the name of reusable component, the name of property certified of the component, the obtained value of certified component should be mentioned, the name of original developer, the name of current owner of the component, status of certificate, date of certification, validity of certification, and the name of CA [9].

\section{2) Issuance of Certificate:}

The certificate will then be issued to the applicant or the one who has requested for certification. The CA will notify the applicant and others concerned about the issuance of certification [9].

\section{3) Publishing of Certificate:}

After issuing the certificate to the applicant, it will be published and will be stored in the database of CA with the concerned details of certificate such as date of issuance, name of issuer, date of expiry, name of certificate holder etc. This is because the CA may notify the applicant about the expiry or renewal of certificate [9].

\section{4) Revoking and Suspension of Certificate:}

If any of the malpractices or discrepancies is found at later stage in the component, which are not stated by developer or kept hidden at the time of certification, the certificate can be revoked or be suspended. The certificate can be revoked or suspended for a specified time interval if mentioned by the CA. Otherwise; it can be revoked or suspended for an infinite time interval also [9].

\section{5) Renewal of Certificate:}

The CA should also provide the renewal services for certification. It should notify the certificate holder before the expiry of certificate, and reminds him of the renewal of certificate [9] [11].

\section{CONCLUSIONS}

The study highlighted the significance of certification of reusable software components. It maintained that software components with valid certificates reflect the quality aspect, whereas the components without certificates are difficult to rely on. To overcome such areas of problem, 
the study stated the certification framework. The stated framework provided the detailed description about the methods, practices, and services related to certification. The methods, practices, and services have been stated after the detailed review of ongoing practices of software security certification. It also noticed the absence of standard certifying authority for quality certification of reusable software components. To overcome this deficiency the study emphasized on the need of certifying authority for quality certification. To curb the need of introducing new certifying authority, it maintained that the existing certifying authorities may club the quality and security certification as and when required.

\section{REFERENCES}

[1] Denney, E., and Fischer, B., (2005), "Software Certification and Software Certificate Management Systems", In Proceedings of the 2005 ASE Workshop on Software Certificate Management.

[2] Wikipedia Contributors, (2017), "Certification”, from web site https://en.wikipedia.org/wiki/Certification accessed on 20/02/2017.

[3] Malik, N., (2013), "Certification of Software Components for Reuse”, International Journal of Advanced Research in Electrical, Electronics and Instrumentation Engineering, 2(9), pp. 4603-4606.
[4] Burger, E., and Reussner, R., (2011), "Performance Certification of Software Components”, Electronic Notes in Theoretical Computer Science, 279(2), pp. 33-41.

[5] GlobalSign, (2016), "GlobalSign Subscriber AgreementVersion 3.5", from web site https://www.globalsign.com/en/repository/globalsignsub scriber-agreement-digital-certificates-and-services.pdf accessed on 25/02/2017.

[6] GlobalSign, (2017a), "GlobalSign Certificate Policy", https://www.globalsign.com/files/3014/8482/1201/Globa ISign_CP_v5.4.pdfaccessed on 25/02/2017.

[7] Shao, Z., (2010), "Certified Software”, Communications of the ACM, 53(2), pp. 56-66.

[8] Wohlin, C., and Runeson, P., (1994), "Certification of Software Components", IEEE Transactions on Software Engineering, 20(6), pp. 494-499.

[9] GlobalSign, (2017b), "GlobalSign CA Certification Practice Statement”, from web site https://www.globalsign.com/files/9414/8482/1137/Globa ISign_CA_CPS_v8.4.pdf accessed on 25/02/2017.

[10] IFPUG, (2014), "Software Certification", from web site http://www.ifpug.org/certification/software-certification/ accessed on 21/02/2017.

[11] GlobalSign, (2017c), "Maximum Certificate Validity”, from web site

[12] https://support.globalsign.com/customer/en/portal/article s/1464693-maximum-certificate-validity accessed on $25 / 02 / 2017$ 